\title{
Rendición de cuentas en la UNA. Metodología: dinámica de los triángulos aplicada por el movimiento ciudadano que construye territorios seguros
}

\section{Accountability of the National University of Costa Rica (UNA). Methodology: Dynamics of Triangles Applied to the Citizen Movement That Builds Safe Spaces}

\author{
Carlos Campos Rojas \\ Universidad de Costa Rica \\ San José, Costa Rica \\ carlosacamposr@gmail.com
}

Recibido: 16/02/2018 Aceptado: 30/05/2018

Resumen. Este trabajo final de graduación, bajo la modalidad de práctica dirigida, se lleva a cabo en la Vicerrectoría de Extensión de la UNA. Visibiliza la gestación de un movimiento ciudadano que hace valer los actuales principios constitucionales de la República de Costa Rica, que acreditan a la persona ciudadana como "El Soberano" y, por ende, como el dueño y depositario todos los días del poder -con atributos pertinentes para el efecto-, algo para lo cual la ciudadanía no ha sido preparada. Por eso, el movimiento lidia con la institucionalidad nacida en el contexto de una república representativa, con estricto poder delegado, en la cual los vicios y costumbres del autoritarismo y de una cultura de la impunidad y la corrupción se asientan. Este trabajo pone en evidencia los esfuerzos de una ciudadanía dialógica, que con su propia metodología construye diariamente, con mucho esfuerzo y con alma de mujer, su propia concepción de mundo, reasumiendo ese poder delegado, como actor del proceso de toma de decisiones Desde un enfoque 
Revista Universidad en Diálogo • Vol. 8, N. 2, Julio-Diciembre, 2018, pp. 39-50

ISSN 2215-2849 • EISSN: 2215-4752

DOI: http://dx.doi.org/10.15359/udre.8-2.3

cualitativo se consideran discusiones y planteamientos teóricos que fundamentan esta práctica dirigida, así como la riqueza de saberes y conocimientos que emanan de la cotidianidad, de la acción metódica de quienes participan en este movimiento que, apropiados de la metodología y sus procedimientos innovadores, propician, en consecuencia, una cultura efectiva de rendición de cuentas y medición de impactos, positivos y negativos.

Para la UNA es especialmente importante dicha experiencia, pues en su última reforma estatutaria el tema de rendición de cuentas, a su propia comunidad y a la "sociedad" ", se tornó en uno de los cambios más relevantes, aunque sin prever un mecanismo que lo haga posible, específicamente, en relación con su rendición a la sociedad y a las comunidades con las que se trabaja.

Palabras claves: El Soberano, poder; miedo, participación en la toma de decisiones, potestad, rendición de cuentas a la sociedad, República Participativa.

Abstract. This graduation work, under the directed practice modality, is carried out in UNA's "Vicerrectoría de Extensión" (Vice Presidency for University Extension). It makes visible the gestation of a citizen movement that enforces the current constitutional principles of the Republic of Costa Rica; these principles empower the citizen as "The Sovereign", and, therefore, as the owner and depositary of everyday power-with the relevant attributes, something for which citizenship has not been prepared. Because of that, the movement deals with the institutionality born in the context of a representative republic with strict delegated power; in this republic are established vices and customs of the authoritarianism and a culture of impunity and corruption. This paper highlights the efforts of dialogical citizenship that, with its own methodology, daily constructs its worldview with great effort and with the woman`s soul, reassuming that delegated power as a key part in the decision-making process. From a qualitative approach are considered discussions, theoretical approaches that support the directed practice, and the rich wisdom and knowledge that emanate from everyday life, as well as from the methodical action of those who take part in this movement, those who, having appropriated the methodology and its innovative procedures, contribute to an effective accountability culture and measurement of impacts-positive and negative ones. For the UNA, this experience is especially important, since in its latest statutory reform the issue of accountability to its own community and "society" became one of the most relevant changes, although, without foreseeing a mechanism to make it possible in relation to the accountability to society and the communities with which the UNA works.

Keywords: The Sovereign, power, fear, participation in decision-making, authority, accountability to society, participatory Republic. 


\section{Introducción}

El motivo de la realización de esta práctica dirigida fue sistematizar los resultados de la aplicación de la metodología de la dinámica de los triángulos, que el Movimiento de Ciudadanía que Construye Territorios Seguros ha venido desarrollando con el fin de sustentar su accionar diario. Este movimiento se ha venido reconociendo y asumiendo como parte activa de "El Soberano", asimismo, se ha venido apropiando de la rendición de cuentas, también consignada en la Constitución Política. No por casualidad comenzaron a construirse en localidades o espacios geográficos fuera del Valle Central.

La cultura que se impone en Costa Rica, desde los comienzos de los años ochenta, amparada en el nuevo modelo económico que regirá el país, propicia en las personas habitantes una distancia con los valores patrios, sus raíces, su historia y sobre todo con el ejercicio y la comprensión de sus derechos políticos. Así, la ciudadanía en general desconoce no solo sus potestades constitucionales o sus atributos ciudadanos, sino, principalmente, en qué consiste el ejercicio de sus derechos políticos y el ejercicio de su poder; también el de sus nuevas responsabilidades consignadas en el contexto de lo que establece nuestra Constitución Política, modificada sustancialmente el 31 de julio del 2003 en su artículo 9, que establece la república participativa y consolida el reconocimiento de la persona, en tanto que ciudadana, como El Soberano. Algo que viene a reforzar el ya importante cambio en el artículo 11, realizado en junio del 2000, que había sentado la obligación constitucional para la persona funcionaria pública-simple depositaria de la autoridad- de la rendición de cuentas y la demostración del impacto de su gestión a la ciudadanía. Estos cambios sutiles, al punto que podrían pasar desapercibidos, implican una evolución sustancial de nuestro régimen político, es decir, en lo relativo al poder, dónde radica y quién lo ejerce.

La institucionalidad nacional, consciente del conflicto de poder que queda así zanjado a favor de la ciudadanía, ha omitido estos cambios y sus consecuencias con un discurso lleno de ambigüedades. El resultado ha sido un descrédito casi anómico por parte de los ciudadanos y las ciudadanas, junto con el incremento de la corrupción y la impunidad en todas las esferas institucionales, no solamente del Estado, sino especialmente en los partidos políticos, las organizaciones gremiales, la Iglesia. Pero la opacidad tiene un costo político. 
En este marco se ha desarrollado la metodología de la dinámica de los triángulos, un instrumento para que la persona ciudadana pueda efectuar su análisis y establecer su participación en la toma de decisiones, cuando desea emprender el camino que lleva a asumir, con todas sus potestades y responsabilidades, la condición constitucional de El Soberano.

El objetivo general de esta sistematización es analizar la metodología llamada la dinámica de los triángulos, aplicada en la Construcción del Movimiento de Ciudadanía que Construye Territorios Seguros, que toma en consideración los artículos 9 y 11 de la Constitución Política de Costa Rica, particularmente en las Actividades de Gala de Rendición de Cuentas y Medición de Impacto del año 2017. Esto en relación con el importante tema de rendición de cuentas a la sociedad que la UNA tiene que resolver.

Para esto se plantean objetivos específicos que orientan el trabajo de esta práctica dirigida, para establecer el conjunto ordenado de acciones que lleva a cabo el Movimiento cuando organiza la Actividad de Gala de Rendición de Cuentas y Medición de Impacto, la que realiza de manera autogestionada. También para reflexionar sobre la forma en la que el Movimiento formula políticas para la participación en la toma de decisiones, bajo su concepción de El Soberano en una república participativa. En otras palabras, cómo las personas integrantes del Movimiento, con sus distintas concepciones, construyen una idea del poder ciudadano que incide en el devenir institucional. Se requiere también conocer los puntos de vista de jerarcas de los poderes de la república, entidades autónomas y empresarios/as. Pero, esencialmente, se trata de validar las conclusiones y recomendaciones con un grupo de la Vicerrectoría de Extensión de la UNA para incidir en su política, aprobada en el III Congreso de Extensión, celebrado en noviembre del 2017.

\section{Metodología}

Aquí se han tomado en cuenta las principales discusiones y planteamientos teóricos que fundamentan esta práctica dirigida, así como la riqueza de saberes y conocimientos que emanan de la práctica, de la acción metódica de quienes participan en este movimiento.

Se adoptó un enfoque cualitativo, en la medida en que es el relato de la vivencia del conflicto de poder que surge entre una institucionalidad que 
lo ha usurpado y una ciudadanía que busca recuperar lo que de por sí le pertenece, proceso en el cual se descubre a sí misma, a través del ejercicio de la potestad constitucional de El Soberano.

Para obtener el resultado de esta práctica se realizaron entrevistas a personas del Movimiento. A esto se sumaron entrevistas y observaciones de las personas participantes, por el método de caso extendido, para recoger el sentimiento y el sentido de valores de aquellos/as que se construyen como El Soberano. Se procesaron también los debates originados por las personas del Movimiento que integran uno de los grupos de WhatsApp. Asimismo, se entrevistó y conoció la opinión de personas o autoridades de los distintos poderes y entidades, así como empresarios/as que tienen una incidencia significativa en el tema. A eso se sumó la consulta a personas que intervinieron en los cambios constitucionales que propiciaron esta apertura hacia el empoderamiento de la ciudadanía. Además, se hizo una identificación representativa de experiencias institucionales que, sobre procesos de rendición de cuentas y transparencia, se llevan en el país, así como de las características de su enfoque, de tal manera que nos permitieran enriquecer la reflexión de esta práctica dirigida. Finalmente, mediante el diálogo con personal de la Vicerrectora de Extensión, se acordó llevar adelante esta práctica dirigida, como un insumo en la toma de decisiones para que la UNA aborde el tema de la rendición de cuentas a la "sociedad", centrada en la peliaguda cuestión del mecanismo, ausente en el Estatuto Orgánico vigente.

\section{Sobre la metodología de la dinámica de los triángulos}

Se trata de una metodología dialéctica que busca que la persona se reconozca primero como sujeto activo, como protagonista o actor principal del proceso, como persona gestora en la construcción de una institucionalidad que responda al mandato constitucional. La persona tiene que deconstruir lo aprendido para establecer su nuevo marco de responsabilidades y deberes, ya que no ha sido educada ni preparada para asumir ese papel.

La metodología es fácil de entender, se construye a partir de una cartulina en blanco, aportada por la persona o las personas interesadas, quienes además son responsables de organizar la reunión. La información que se plasma en la cartulina es, fundamentalmente, la percepción, los saberes y los conocimientos de las personas asistentes. Así se logra que la metodología termine siendo no solo el resultado de un trabajo conjunto, sino también saber que sus palabras y criterios empiezan a tener un sentido. 
Revista Universidad en Diálogo • Vol. 8, N. 2, Julio-Diciembre, 2018, pp. 39-50

ISSN 2215-2849 • EISSN: 2215-4752

DOI: http://dx.doi.org/10.15359/udre.8-2.3

El objetivo principal es reconocer lo determinante del yo persona, como sujeto transformador y el descubrimiento de un nuevo nosotros/as, formado por muchos yo, todos con palabra y decisión, en las mismas condiciones, pues la jerarquía y la representación no tienen cabida en esta metodología, ni en la herramienta de construcción de organización, es decir, la relación es de sujeto activo a sujeto activo y no de sujeto activo a sujeto pasivo, que se transforma en objeto. No se trata de establecer una nueva relación asimétrica de dominación de unas personas sobre otras, sino de reconocer que su poder solo se activa a partir de su autodeterminación y no como sujeto de la aprobación de otros/as.

Son tres los compromisos que cada persona debe asumir consigo misma. El empoderamiento es así el primer compromiso insoslayable. En segundo lugar, se trata de vencer el miedo, primero el más significativo: el miedo al yo interno, aquel que paraliza a la persona, que le impide asumir los procesos de cambio y transformación, el que la somete en el ámbito social, el que la mantiene bajo control social, el que se asienta en la colonialidad del poder, de un poder que se percibe externo y omnipotente. Además, enfrentar el miedo a la institucionalidad y los/as funcionarios/as públicos/as.

Está absolutamente prohibido "pedir" y hablar recurriendo al uso de esta palabra, dada la connotación de sumisión y dependencia que se le ha conferido, principalmente desde un Estado asistencialista y con énfasis en el clientelismo. La metodología establece el deber como la norma ciudadana originaria y reconoce que el éxito del proceso está determinado por la convicción que asume la persona, pues se requiere solo una para fundar un territorio seguro.

Se refuerza el concepto de la persona que ahora es protagonista del proceso, otorgándose y asumiendo el respeto y el reconocimiento a sí misma que antes no tenía, pues en el nosotros/as construido hasta ahora la persona, el yo, es invisible, sujetada, determinada y limitada por otros/as, adecuadamente construida como parte del control social.

La metodología ofrece a la persona la posibilidad de adquirir conocimiento, de desempeñarse mejor de manera constante, pues su responsabilidad constitucional le exige intervenir en la toma de decisiones para fijar el norte nacional. No tiene que esperar a otro/a, ni depende de eso para actuar; es sujeto no objeto; tampoco está determinada a la legitimación del/de la otro/a, para eso aprende a reconstruir su poder y entender cómo aplicarlo. 
Para el tercer compromiso se propone asumir y desarrollar la cultura de rendición de cuentas y la medición de impacto, pues ahora tiene conciencia de la existencia de El Soberano y su responsabilidad constitucional, por lo que le corresponde conocer el destino de la inversión del pago de sus impuestos, que muchas veces aporta a costa de su vulnerabilidad y su carencia de oportunidades, para que la empresa más importante de la que es dueño/a funcione: el país.

La metodología de la dinámica de los triángulos (figura 1) construye así un nuevo tejido social, que tiene que ser dialógico entre sí y para sí, donde la persona se propone construir y descubrir -con concepción de poder- los puentes para acercarse a la institucionalidad y a sus funcionarios y funcionarias, al empresariado y a la ciudadanía, para ejercer el poder que le corresponde constitucionalmente. Aprende a dimensionar la confrontación, la guerra ("resistencias", protestas, quejas, huelgas, paros, manifestaciones, etc.) para desarrollar un blindaje de su proceso, rodeado de inflexiones para rehacerse de su poder ciudadano.

\section{CONSTRUIR TERRITORIOS SEGUROS... UN CONCEPTO DE VIDA}

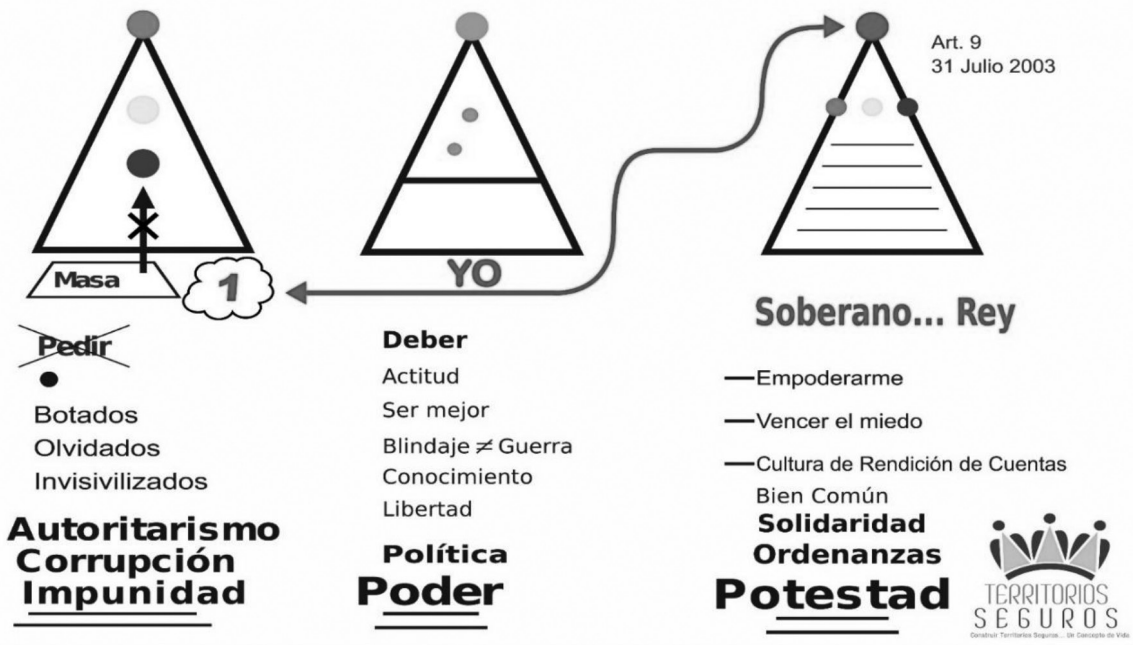

Figura 1. Metodología de la dinámica de los triángulos

Fuente: elaboración propia 
De esta forma, la persona aprende a demostrar su capacidad dialógica, la habilidad para construir consensos, concebidos como la capacidad de construir acuerdos (Dussel, 2006) a partir de las diferencias, superar el miedo de la ciudadanía restringida; a reconocerse con la habilidad para el debate y la propuesta, sumando las voluntades necesarias para construir una institucionalidad que restituya la gobernabilidad perdida, que hoy se debate ante la negación de quienes se aferran exclusivamente a la representatividad y al poder delegado.

Este modelo facilita enfrentar las disensiones interpersonales o intergrupales en el seno del Movimiento, pues donde existen y no parecen resolverse se encuentra la posibilidad de separarse y continuar su propio proceso, poniendo a prueba sus puntos de vista, su práctica y su interpretación de la realidad, desde su autónoma organización: el territorio seguro. Así, la libertad, y lo que ella significa, es un valor sumamente preciado en el proceso.

\section{Algunos resultados}

La herramienta de organización que se utiliza se denomina construcción de territorio seguro, el modelo se ha venido poniendo en práctica desde octubre del 2009, modelo que desarrollé a partir de integrar diferentes experiencias organizativas anteriores, en las que tuve participación directa. Se rompen los esquemas, no es necesario contar con personería jurídica, hacerlo es restringir las potestades constitucionales asignadas a la persona y limitar su poder. Hoy se construyen más de cuatrocientos territorios seguros en todo el país, en veinte cantones.

Ahora ya se cuenta con suficientes datos (ver cuadro 1) que demuestran la fortaleza de la metodología en los procesos de rendición de cuentas, sin embargo, es mejor que lo exprese una compañera ama de casa:

Los Triángulos son como el abecedario, cada letra está predeterminada con un sonido, pero la unión de ciertas letras va a formar una palabra ... con los elementos que ahorita tiene, yo puedo entender, construir, desarrollar y entender "si de verdad es lo que deseo", cómo construir el proceso que me va a beneficiar, que me va a hacer crecer, que me va a convertir en ese Yo Soberano, que va a ejercer la autoridad de una persona con conocimiento, para tener un control del Poder de una manera equitativa, de manera que beneficie a mi persona, y también a muchos (Zúñiga, 2017). 
Cuadro 1

Actividades y aplicaciones metodológicas realizadas

\begin{tabular}{|c|c|c|c|}
\hline & $\begin{array}{l}\text { Ene-Oct } \\
2017\end{array}$ & $\begin{array}{c}\text { Ene-Dic } \\
2016\end{array}$ & Oct 2009-Dic 2015 \\
\hline Actividades de gala realizadas & 3 & 3 & 13 \\
\hline Cantones realizando actividades de gala & 2 & 2 & 1 \\
\hline Memorias procesadas & 3 & 2 & 1 \\
\hline $\begin{array}{l}\text { Presentación de metodología en } \\
\text { territorios seguros }\end{array}$ & 22 & 74 & 387 \\
\hline $\begin{array}{l}\text { Acompañamiento para aplicar la } \\
\text { metodología }\end{array}$ & 55 & 153 & 426 \\
\hline $\begin{array}{l}\text { Entrevistas a personas de territorios } \\
\text { seguros }\end{array}$ & 29 & & \\
\hline $\begin{array}{l}\text { Entrevistas a jerarcas institucionales } \\
\text { y personas empresarias }\end{array}$ & 14 & & \\
\hline $\begin{array}{l}\text { Análisis de contenido de grupo de } \\
\text { WhatsApp }\end{array}$ & 414 & & \\
\hline $\begin{array}{l}\text { Publicaciones realizadas (congresos, } \\
\text { periódicos, etc.) }\end{array}$ & 8 & 6 & 8 \\
\hline $\begin{array}{l}\text { Talleres de Evaluación y } \\
\text { Planeamiento realizados }\end{array}$ & 24 & 15 & 40 \\
\hline $\begin{array}{l}\text { Cantones visitados atendiendo } \\
\text { invitaciones }\end{array}$ & 10 & 17 & 8 \\
\hline $\begin{array}{l}\text { Comunidades visitadas atendiendo } \\
\text { invitaciones }\end{array}$ & 115 & 145 & 232 \\
\hline
\end{tabular}

Fuente: elaboración propia.

En Pococí, donde nace el Movimiento de Ciudadanía que Construye Territorio Seguro, se instala la Actividad de Gala de Rendición de Cuentas y Medición de Impacto. El 20 de octubre de este año se celebra la decimoquinta actividad, la cual se realiza dos veces por año. El poder de convocatoria desarrollado por la ciudadanía que organiza la actividad 
Revista Universidad en Diálogo • Vol. 8, N. 2, Julio-Diciembre, 2018, pp. 39-50

ISSN 2215-2849 • EISSN: 2215-4752

DOI: http://dx.doi.org/10.15359/udre.8-2.3

ya reúne más de setenta instituciones, lo que demuestra la madurez y el poder que han ido logrando, así como la seriedad y responsabilidad con que asumen el proceso. En Sarapiquí comenzaron el año pasado y el 27 de octubre se estará realizando ya el segundo evento.

La metodología ha sido puesta a prueba en diferentes partes del país, así como en segmentos sociales, económicos, políticos, académicos diversos, demostrando la consistencia de la misma. Con ella, se construye una ciudadanía autónoma desde la misma ciudadanía, con el deber como norte y la actitud permanente de mantener su paso. Ella permite desarrollar la capacidad para empezar a incidir en transformar la cultura institucional de una república representativa, que se ha venido sustentando en el autoritarismo y que se nutre de la corrupción y la impunidad. Así, se abre paso una ciudadanía que se prepara descubriendo y desarrollando destrezas y capacidades "que el Estado y las instancias tradicionales de participación ciudadana le han negado", para sustentar otra institucionalidad, la que establece nuestra Constitución Política: la institucionalidad de la república participativa, sin la cual la representativa tampoco podrá mantenerse, pues la persona representante solo representa cuando quien delegó la representación mantiene sus potestades.

\section{Recomendación de un mecanismo de rendición de cuentas de la UNA para la sociedad}

Esta experiencia ciudadana, que emerge desde la ciudadanía misma como una oportunidad para una nueva gobernanza participativa, puede iluminar la búsqueda de un mecanismo para que la UNA resuelva su propio desafío no solo de rendir cuentas a la sociedad, sino de construir ciudadanía desde lo interno. Este podría ser un proceso inducido inicialmente por convocatoria de la UNA, donde existe la determinación institucional de insertarse en el entorno donde se asienta y reconoce como parte suya la ciudadanía que habita el área de impacto institucional. Esto dará pie para la formación de tres Consejos Ciudadanos para la Transparencia Institucional (CCTI), uno para cada acción sustantiva (más un cuarto Consejo que se especificará al final), donde mujeres, jóvenes y hombres participen con equidad, deseo, criterio y responsabilidad de acceder a formarse para cumplir con la función y la tarea encomendada.

El Consejo del área de docencia podría constituirse por un grupo de madres y padres de familia con hijos/as becados/as o no, por ejemplo. El del área 
de investigación por personas usuarias no universitarias, donde se integren participantes de los procesos de los/as mismos/as investigadores/as. El del área de extensión por un grupo de participantes no universitarios/as en los proyectos de extensión, que surgen de quienes habitan el entorno de esos proyectos, especialmente y no esencialmente, como es la tradición, lo fácil, la costumbre, exclusivamente por líderes comunitarios/as y de organizaciones de sociedad civil supuestamente representativos.

En las Sedes Regionales se pueden constituir esos Consejos a partir de la vieja figura de los Consejos de Vinculación, presente en el Reglamento de Sedes Regionales $-\mathrm{y}$ que no se ha puesto en práctica-, provocando procesos de acercamiento e interacción con las personas y comunidades del entorno, con el decidido empeño de crear conjuntamente una cultura de participación ciudadana en la toma de decisiones y de rendición de cuentas, sin exclusiones ni aislamientos, lo que provocará la incorporación de personas desde otro paradigma, en las tres áreas sustantivas de docencia, investigación y extensión.

Con cada uno de esos CCTI la UNA tendría que establecer una serie de compromisos a dos años plazo, rindiendo cuentas en esos mismos plazos, publicando los resultados de esas sesiones y procesos de rendición de cuentas, para que la sociedad crezca y la universidad lo haga con ella. Una vez constituidos - de acuerdo con su propio modelo de extensión-, la UNA motivará su desarrollo autónomo, para que adopten su propia normativa, así como los procedimientos y los recursos para autoconvocarse y renovarse.

También podría pensarse, alternativamente, en un solo CCTI conformado de manera mixta, según se ha indicado, para recibir cuentas de las tres acciones sustantivas, tal como se sugiere para cada Sede Regional. Asimismo, podría comenzarse con el Consejo del área de extensión, a modo de una experiencia piloto. Para empezar, serían instancias ad hoc y después de un tiempo podrían ser parte de la estructura, según fuera aprobada la reforma estatutaria del caso. Para que esto ocurra habrá que ir trenzando un mecate de dos puntas: la voluntad política de la UNA y la voluntad de participación e incidencia de sus personas aliadas más cercanas. Sin embargo, la primera de ellas tendrá al principio la voz cantante y confiamos en que en Extensión existe la reserva ética para impulsarlo y supervisarlo. Sarapiquí es ya un punto de partida significativo, pero aún opaco para la institución y carente de atención. 
Las personas que los constituyan se desarrollarán, a su vez, como una ciudadanía soberana y participante, aprovechando la metodología de la dinámica de los triángulos para ello. Las personas participantes más destacadas podrían constituir un Consejo Social de Rectoría, también ad hoc, que se ocuparía de revisar y dar seguimiento a los acuerdos tomados con los CCTI, a lo largo de los dos años, según la idea que propusiera, al inicio de su gestión, el actual rector.

Esta propuesta se hace con el fin de iniciar un debate, que en la UNA no se ha podido empezar todavía en ninguna instancia y por ninguna persona. Espero que dé "el pie", como se dice en teatro, para una reflexión que conduzca a establecer un mecanismo eficaz que enriquezca las políticas de la UNA y la ponga a la vanguardia de la transparencia que la nueva institucionalidad universitaria requiere en la nueva república participativa, que espera su puesta en práctica desde el 2000 y el 2003.

\section{Referencias}

Dussel, E. (2006). Tesis de política. México: Editorial Siglo XXI.

Zúñiga, K. (Miembro del Territorio Seguro Brisas 2). (1 de marzo de 2017). Participación en debate del grupo de WhatsApp: Territorio Seguro Oficina, 12:05 p.m. 